\title{
Flavor changing top decays to charm and a Higgs boson with $\tau \tau$ at the LHC
}

\author{
Phillip Gutierrez $\odot,{ }^{*}$ Rishabh Jain $\odot,{ }^{\dagger}$ and Chung Kao ${ }^{\ddagger}$ \\ Homer L. Dodge Department of Physics and Astronomy, University of Oklahoma, \\ Norman, Oklahoma 73019, USA
}

(Received 20 March 2021; accepted 10 May 2021; published 15 June 2021)

\begin{abstract}
We investigate the prospects of discovering the top-quark decay into a charm quark and a Higgs boson $\left(t \rightarrow c h^{0}\right)$ in top-quark pair production at the CERN Large Hadron Collider (LHC). A general twoHiggs-doublet model is adopted to study flavor-changing neutral Higgs (FCNH) interactions. We perform a parton-level analysis as well as Monte Carlo simulations using Pythia 8 and Delphes to study the flavor-changing top-quark decay $t \rightarrow c h^{0}$, followed by the Higgs decaying into $\tau^{+} \tau^{-}$, with the other top quark decaying to a bottom quark $(b)$ and two light jets $(t \rightarrow b W \rightarrow b j j)$. To reduce the physics background to the Higgs signal, only the leptonic decays of tau leptons are considered, $\tau^{+} \tau^{-} \rightarrow e^{ \pm} \mu^{\mp}+E_{T}$, where $E_{T}$ represents the missing transverse energy from the neutrinos. In order to reconstruct the Higgs boson and top-quark masses as well as to reduce the physics background, the collinear approximation for the highly boosted tau decays is employed. Furthermore, the energy distribution of the charm quark helps set the acceptance criteria used to reduce the background and improve the statistical significance of the signal. We study the discovery potential for the FCNH top decay at the LHC with collider energy $\sqrt{s}=13$ and $14 \mathrm{TeV}$ as well as a future hadron collider with $\sqrt{s}=27 \mathrm{TeV}$. Our analysis suggests that a high-energy LHC at $\sqrt{s}=27 \mathrm{TeV}$ will be able to discover this FCNH signal with an integrated luminosity $\mathcal{L}=3 \mathrm{ab}^{-1}$ for a branching fraction $\mathcal{B}\left(t \rightarrow c h^{0}\right) \gtrsim 1.4 \times 10^{-4}$, which corresponds to a FCNH coupling $\left|\lambda_{t c h}\right| \gtrsim 0.023$. This FCNH coupling is significantly below the current ATLAS combined upper limit of $\left|\lambda_{t c h}\right|=0.064$.
\end{abstract}

DOI: $10.1103 /$ PhysRevD.103.115020

\section{INTRODUCTION}

The discovery of the Higgs boson in 2012 [1,2] completed the experimental observation of the particle spectrum predicted by the Standard Model (SM). A primary goal of the high-luminosity and higher-energy Large Hadron Collider (LHC) is the precision testing of the SM and the search for physics beyond the Standard Model (BSM), especially the interactions of the Higgs boson, the top quark, and sources of $C P$ violation. Several experimental searches [3-6] are being performed to improve the understanding of Higgs boson interactions with SM particles and to search for possible extensions of the Higgs sector.

There are some deviations from the SM such as the presence of baryon asymmetry in the Universe [7] requiring

\footnotetext{
pgutierrez@ou.edu

†ishabh.Jain@ou.edu

ॠChung.Kao@ou.edu
}

Published by the American Physical Society under the terms of the Creative Commons Attribution 4.0 International license. Further distribution of this work must maintain attribution to the author(s) and the published article's title, journal citation, and DOI. Funded by SCOAP ${ }^{3}$.
$C P$ violation beyond that predicted by the SM. The muon anomalous magnetic moment measurements at BNL [8] and Fermilab [9] show an approximately $4.2 \sigma$ deviation from the SM [10-12]. In addition, there might be possible flavor anomalies among the quarks and leptons [13-17]. The Standard Model with one Higgs doublet cannot explain these anomalies [18], thus requiring BSM physics. A general two-Higgs-doublet model (2HDM) provides a simple extension to the SM. It consists of two scalar SU(2) doublets, which after electroweak symmetry breaking (EWSB) lead to five physical Higgs bosons: two $C P$-even scalars $\left[H^{0}\right.$ (heavier) and $h^{0}$ (lighter)], one $C P$-odd pseudoscalar $\left(A^{0}\right)$, and a pair of charged Higgs bosons $\left(H^{ \pm}\right)$. General 2HDMs can provide additional sources of $C P$ violation $[19,20]$, and generate tree-level flavorchanging neutral Higgs (FCNH) interactions that can enhance the branching fractions of flavor-changing neutral currents, especially $t \rightarrow c \phi^{0}$ [21], $\phi^{0} \rightarrow t \bar{c}$ or $\bar{t} c$ [22,23], and $\phi^{0} \rightarrow \tau^{ \pm} \mu^{\mp}$ [24-27], where $\phi^{0}=H^{0}, h^{0}$, and $A^{0}$. The $\mathrm{SM}$ expectation is $\mathcal{B}\left(t \rightarrow c h^{0}\right) \approx 10^{-14}$ [28-30], which is significantly less than current and near-term experiments can observe. If the FCNH signal $t \rightarrow c h^{0}$ is observed at the LHC or HL-LHC, it would imply BSM physics [31-44].

We adopt the Yukawa Lagrangian in a general twoHiggs-doublet model $[45,46]$ as 


$$
\begin{aligned}
\mathcal{L}_{Y}= & \frac{-1}{\sqrt{2}} \sum_{F=U, D, L} \bar{F}\left\{\left[\kappa^{F} s_{\beta-\alpha}+\rho^{F} c_{\beta-\alpha}\right] h^{0}\right. \\
& \left.+\left[\kappa^{F} c_{\beta-\alpha}-\rho^{F} s_{\beta-\alpha}\right] H^{0}-i \operatorname{sgn}\left(Q_{F}\right) \rho^{F} A^{0}\right\} P_{R} F \\
& -\bar{U}\left[V \rho^{D} P_{R}-\rho^{U^{\dagger}} V P_{L}\right] D H^{+}-\bar{\nu}\left[\rho^{L} P_{R}\right] L H^{+}+\text {H.c. },
\end{aligned}
$$

where $P_{L, R} \equiv\left(1 \mp \gamma_{5}\right) / 2, \quad c_{\beta-\alpha} \equiv \cos (\beta-\alpha), \quad s_{\beta-\alpha} \equiv$ $\sin (\beta-\alpha), \alpha$ is the mixing angle between neutral Higgs scalars, $\tan \beta \equiv v_{2} / v_{1}$ [47] is the ratio of the vacuum expectation values of the two Higgs doublets, $Q_{F}$ is the fermion charge, and the $\kappa$ matrices are diagonal and fixed by fermion masses to $\kappa^{F}=\sqrt{2} m_{F} / v$, with $v \approx 246 \mathrm{GeV}$, while the matrices $\rho$ contain both diagonal and off-diagonal elements with free parameters. In addition, $F, U, D, L$ represent elementary fermions, up-type quarks, down-type quarks, and charged leptons, respectively. The matrix elements $\rho$ are the FCNH couplings to the fermions. Almost all experimental data are consistent with the Standard Model [48,49], which implies that all twoHiggs-doublet models must be in the decoupling [50] or alignment limit [51,52] with one SM-like light scalar $\left(h^{0}\right)$ that has a mass of $125 \mathrm{GeV}$.

Recently, the ATLAS Collaboration [40] combined several channels to search for $t \rightarrow c h^{0}$ with $h^{0} \rightarrow b \bar{b}, h^{0} \rightarrow \tau \tau$ with at least one hadronic tau decay, $h^{0} \rightarrow W W^{*}, Z Z^{*}, \tau^{+} \tau^{-}$ (same sign $2 \ell, 3 \ell$ ), and $h^{0} \rightarrow \gamma \gamma$, and put a strong constraint on the branching fraction $\mathcal{B}\left(t \rightarrow c h^{0}\right) \leq 1.1 \times 10^{-3}$. This leads to an upper limit on the FCNH Yukawa coupling $\left|\lambda_{t c h}\right|$

$$
\lambda_{\text {tch }} \leq 0.064
$$

for the effective Lagrangian

$$
\mathcal{L}=-\frac{\lambda_{t c h}}{\sqrt{2}} \bar{c} t h^{0}+\text { H.c. },
$$

with the relation between $\lambda_{\text {tch }}$ and the $t \rightarrow c h^{0}$ branching fraction [53] being

$$
\lambda_{\text {tch }} \approx 1.92 \times \sqrt{\mathcal{B}\left(t \rightarrow c h^{0}\right)} .
$$

In this article, we investigate the discovery potential of the top-quark decay into a charm quark and a Higgs boson $\left(t \rightarrow c h^{0}\right)$ followed by the Higgs boson decaying into $\tau^{+} \tau^{-}$in top-quark pair production at the CERN LHC. To investigate the discovery potential of a flavorchanging neutral Higgs boson signal with low physics background, we consider only the leptonic decays of the tau leptons, $\tau^{+} \tau^{-} \rightarrow e^{ \pm} \mu^{\mp}+\mathbb{E}_{T}$, where $\mathbb{E}_{T}$ is the missing transverse energy in the event from the neutrinos. This is complementary to the ATLAS searches for same-charge dileptons.
We perform a parton-level analysis as well as a Monte Carlo simulation using Pythia 8 [54] and Delphes [55] to study the FCNH decay of one top quark while the other top quark decays hadronically to a bottom quark $(b)$ and two light jets: $p p \rightarrow t \bar{t} \rightarrow b W^{ \pm} c h^{0} \rightarrow b j j c \tau^{+} \tau^{-}+X$. We calculate the production rates using the full treelevel matrix elements including the Breit-Wigner resonance for both signal and background processes. In addition, we optimize our acceptance using a standard selectionbased technique, as well as using a boosted decision tree to improve the signal-to-background ratio and statistical significance.

Since we do not apply charm tagging, our analysis is suitable for a general search for $t \rightarrow q h^{0}, q=u, c$. Many previous studies have adopted the Cheng-Sher ansatz [56] as the benchmark Yukawa coupling,

$$
\lambda_{t q h}=\frac{\sqrt{2 m_{t} m_{q}}}{v},
$$

where $q=u, c$ and $v \approx 246 \mathrm{GeV}$ is the Higgs vacuum expectation value. The FCNH couplings as the geometric mean for top and charm quarks is

$$
\lambda_{t c h}(C S)=\frac{\sqrt{2 m_{t} m_{c}}}{v} \approx 0.0895
$$

which has been excluded by a recent ATLAS experiment [40]. For simplicity, we assume $\lambda_{t c h} \gg \lambda_{t u h}$ and focus on the search for $t \rightarrow c h^{0}$. To verify that the associated quark is a charm, we will need to apply charm tagging.

There are several aspects to note in this analysis. To reconstruct the Higgs boson and the top quark, the collinear approximation of tau decays [57] is used. The collinear approximation for tau decays with physical momentum fractions $x_{i}\left(0<x_{i}<1\right)$, where $x_{i}=p\left(\ell_{i}\right) / p(\tau), i=1,2$, more effectively reduces the physics background than the centrality requirement suggested in Refs. [37,40]. Furthermore, the energy of the charm quark in the topquark rest frame provides good acceptance for the FCNH top signal while rejecting background [32,58]. Promising results are presented for the LHC with $\sqrt{s}=14 \mathrm{TeV}$ and $27 \mathrm{TeV}$.

\section{HIGGS SIGNAL AND EVENT SELECTIONS}

This section presents the cross section for the FCNH signal $t \rightarrow c h^{0}$ from top-quark pair production and outlines our search strategy for this signal at the LHC. We focus on the discovery channel with one top quark decaying hadronically $(t \rightarrow b j j)$, while the other top quark decays into a charm quark and a Higgs boson $\left(h^{0}\right)$ followed by $h^{0} \rightarrow \tau^{+} \tau^{-} \rightarrow e^{ \pm} \mu^{\mp}+\mathbb{E}_{T}$. Unless explicitly specified, $q$ generally denotes a quark $(q)$ or an antiquark $(\bar{q})$ and $\ell^{ \pm}$ will represent an $e^{ \pm}$or $\mu^{ \pm}$. This means that our FCNH 
signal has the final state of $p p \rightarrow t \bar{t} \rightarrow b j j c e^{ \pm} \mu^{\mp}+$ $E_{T}+X$, where $X$ represents all other particles produced in the $p p$ collision. Since the mass of the Higgs boson is much greater than the tau lepton's mass $\left(M_{h} \gg m_{\tau}\right)$, the tau leptons are highly boosted. Therefore, the collinear approximation of the tau decay [57] is employed to reconstruct the Higgs boson mass and the top-quark mass.

At the parton level, our analysis employs MadGraph5aMC-NLO [59] to calculate tree-level cross sections for the full process $p p \rightarrow t \bar{t} \rightarrow b j j \bar{c} h^{0} \rightarrow b j j \bar{c} \tau^{+} \tau^{-}+X$ along with the collinear approximation of tau decays [57]. The parton-level cross section is evaluated using the CT14LO parton distribution functions (PDFs) [60]. For simplicity, the factorization scale $\left(\mu_{F}\right)$ and the renormalization scale $\left(\mu_{R}\right)$ are chosen to be the invariant mass of the top-quark pair $\left(M_{t \bar{t}}\right)$. With the above scale choices and PDFs, our current estimates suggest a $K$-factor of $\approx 1.8$, which is approximately the same for all three energies $(\sqrt{s}=13,14$, and $27 \mathrm{TeV})$ investigated for top-quark pair production at the LHC. The $K$-factor is calculated using $\mathrm{TOP}++[61]$.

This analysis employs the full tree-level matrix elements to evaluate the cross section for the FCNH signal and physics background. In addition, a consistency check for the tree-level signal cross section has been performed in the narrow-width approximation by calculating the cross section $\sigma\left(p p \rightarrow t \bar{t} \rightarrow t c h^{0} \rightarrow b j j c \ell_{1}^{ \pm} \ell_{2}^{\mp} E_{T}+X\right)$ as the product of cross section times branching fractions:

$$
\begin{gathered}
\sigma(p p \rightarrow t \bar{t} \rightarrow b j j \bar{t}+X) \times \mathcal{B}\left(\bar{t} \rightarrow \bar{c} h^{0}\right) \times \mathcal{B}\left(h^{0} \rightarrow \tau^{+} \tau^{-}\right) \\
\times \mathcal{B}\left(\tau^{+} \rightarrow \ell_{1}^{+} \nu_{\ell_{1}} \bar{\nu}_{\tau}\right) \times \mathcal{B}\left(\tau^{-} \rightarrow \ell_{2}^{-} \bar{\nu}_{\ell_{2}} \nu_{\tau}\right) .
\end{gathered}
$$

To evaluate the branching fraction of $t \rightarrow c h^{0}$, the effective Lagrangian in Eq. (3) is employed. The resulting decay width is then obtained as

$$
\begin{aligned}
\Gamma\left(t \rightarrow c h^{0}\right)= & \frac{\left|\lambda_{t c h}\right|^{2}}{32 \pi} \times\left(m_{t}\right) \times\left[\left(1+r_{c}\right)^{2}-r_{h}^{2}\right] \\
& \times \sqrt{1-\left(r_{h}+r_{c}\right)^{2}} \sqrt{1-\left(r_{h}-r_{c}\right)^{2}},
\end{aligned}
$$

with $r_{h}=M_{h} / m_{t}$ and $r_{c}=m_{c} / m_{t}$. Assuming that the total decay width of the top quark is

$$
\Gamma_{t}=\Gamma(t \rightarrow b W)+\Gamma\left(t \rightarrow c h^{0}\right),
$$

the branching fraction of $t \rightarrow c h^{0}$ is

$$
\mathcal{B}\left(t \rightarrow c h^{0}\right)=\frac{\Gamma\left(t \rightarrow c h^{0}\right)}{\Gamma_{t}} .
$$

Comparing this with the Yukawa Lagrangian in Eq. (1), we can express

$$
\lambda_{t c h}=\tilde{\rho}_{t c} \cos (\beta-\alpha)
$$

with

$$
\tilde{\rho}_{t c}=\sqrt{\frac{\left|\rho_{t c}\right|^{2}+\left|\rho_{c t}\right|^{2}}{2}} .
$$

To present the results, the more convenient free parameters $\tilde{\rho}_{t c}$ and $\cos (\beta-\alpha)$ are chosen for the FCNH Yukawa couplings.

In the event-level analysis, parton-level samples are generated from MadGraph using TauDecay-UFO [62] to model $\tau$ decays, and then the sample is processed with Pythia 8 [54] and Delphes [55] to generate events with hadronization, showering, and detector effects. In addition, the MLM-matching/merging [63] algorithm is applied to match the additional hadronized jets in each event with partons to avoid double counting jets that are generated by parton showering from final-state radiation for all background processes.

To provide a realistic estimate for production rates at the LHC, we evaluate the cross section for the FCNH signal and physics background in $p p$ collisions with the proper tagging and mistagging efficiencies. The ATLAS tagging efficiencies [64] are adopted to evaluate the cross section for the FCNH signal and physics background. The $b$-tagging efficiency is 0.7 , the probability that a $c$ jet is mistagged as a $b$ jet $\left(\epsilon_{c}\right)$ is approximately 0.14 , while the probability that a light jet $(u, d, s, g)$ is mistagged as a $b$ jet $\left(\epsilon_{j}\right)$ is 0.01 .

\section{A. Event selections}

Our FCNH signal comes from top-quark pair production with one top quark decaying into a charm quark and a Higgs boson while the other top quark decays to an allhadronic final state. Every event is required to contain at least four jets, including exactly one that is identified as a $b$ jet. In addition, there are two opposite-charge leptons of different flavor $\left(e^{ \pm} \mu^{\mp}\right)$ with missing transverse energy from neutrinos.

We adopt the following basic requirements, which are similar to the ATLAS and CMS $h^{0} \rightarrow \tau^{+} \tau^{-}$studies [65].

(i) Four jets including one $b$ jet with $P_{T}(b, j) \geq$ $20 \mathrm{GeV}$.

(ii) $|\eta(b, j)| \leq 2.5$.

(iii) Two opposite-charge leptons with $P_{T}(\ell) \geq 10 \mathrm{GeV}$, and $|\eta(\ell)| \leq 2.5$.

(iv) $P_{T}$ (leading $\left.\ell\right) \geq 20 \mathrm{GeV}$.

(v) $\Delta R(\ell \ell, j j, b j, \ell j, \ell b) \geq 0.4$.

(vi) $\mathscr{E}_{T} \geq 25 \mathrm{GeV}$.

(vii) All events containing more than one tagged $b$ jet with $P_{T} \geq 20 \mathrm{GeV}$ and $|\eta|<2.5$ are rejected.

Since the $b$-quark jet is selected through tagging, this leaves three jets to be identified as two light-flavor jets and a $c$-quark jet. The two light-flavor jets, $j_{1} j_{2}$, are selected by minimizing $\left|M_{j j}-m_{W}\right|$ and $\left|M_{b j j}-m_{t}\right|$. The remaining jet is labeled as the $c$-quark jet. For the event to be correctly reconstructed, $j_{1}$ and $j_{2}$ must result from the decay of a $W$ boson, and therefore their invariant mass 

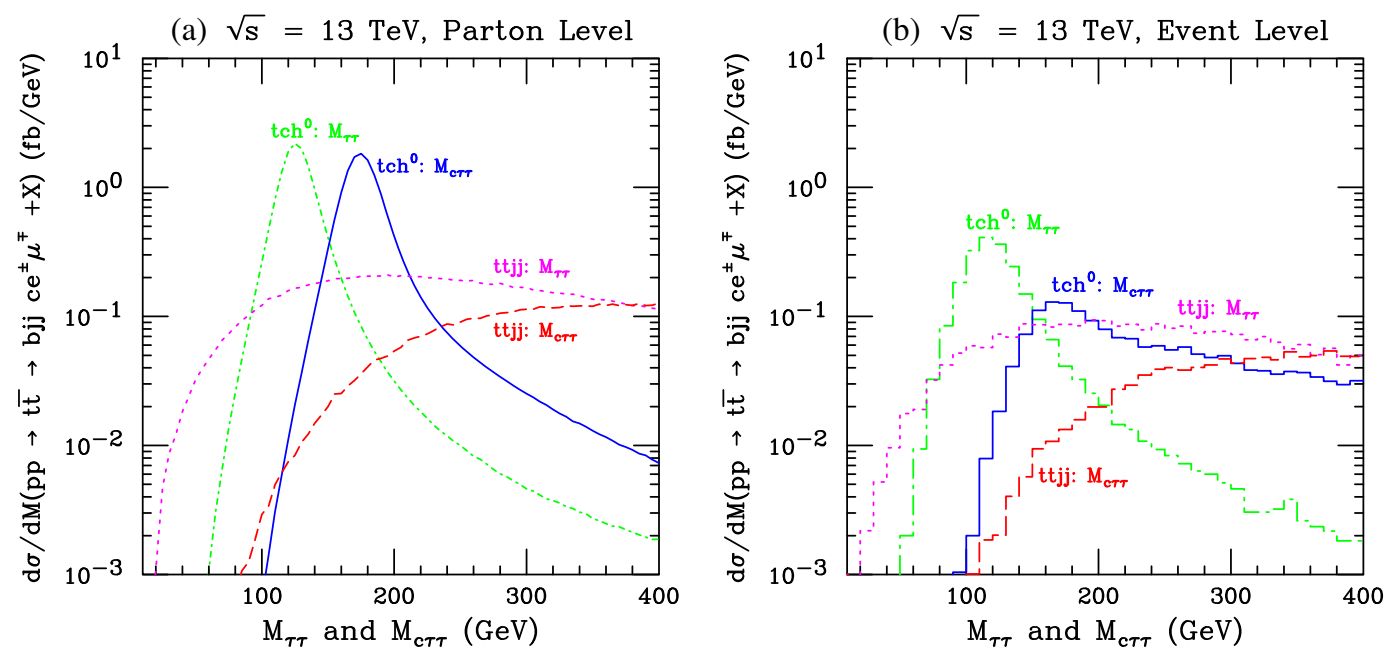

FIG. 1. Invariant mass distributions $d \sigma / d M_{\tau \tau}$ (green dotted dashed) and $d \sigma / d M_{c \tau \tau}$ (blue solid) for the FCNH signal $\left(t \rightarrow c h^{0}\right)$ at the (a) parton level and (b) event level with detector simulation in $p p$ collisions. Also shown are the invariant mass distributions $d \sigma / d M_{\tau \tau}$ (magenta dotted) and $d \sigma / d M_{c \tau \tau}$ (red dashed) for the dominant background from $t t j j$.

distribution $M_{j_{1} j_{2}}$ peaks at $M_{W} \approx 80.4 \mathrm{GeV}$ and $M_{b j_{1} j_{2}}$ has a peak at $m_{t} \approx 173.2 \mathrm{GeV}$. Using the ATLAS mass resolution [66], the reconstructed $W$ and top-quark masses are required to lie in the mass windows $\Delta M_{j_{1} j_{2}}=0.20 M_{W}$ and $\Delta M_{b j_{1} j_{2}}=0.25 m_{t}$.

\section{B. Higgs mass reconstruction}

For the FCNH signal, $t \rightarrow c h^{0} \rightarrow c \tau^{+} \tau^{-} \rightarrow c e^{ \pm} \mu^{\mp}+$ $\mathbb{E}_{T}$, the reconstruction is performed two ways: (a) using the invariant $\tau^{+} \tau^{-}$mass from the Higgs decay and the invariant mass of $c \tau^{+} \tau^{-}$from the top-quark decay, which have sharp peaks near $M_{H}$ and $m_{t}$, and (b) as in Ref. [41], using the cluster transverse masses of $\ell^{+} \ell^{-}$and $c \ell^{+} \ell^{-}$, which have broad peaks near $M_{H}$ and $m_{t}$.

The Higgs boson mass can be reconstructed by applying the collinear approximation [67-69] to the $\tau$ decay products

$$
p_{\ell_{i}}=x_{i} p_{\tau_{i}}, \quad i=1,2 \text { with } p_{T}\left(\ell_{1}\right)>p_{T}\left(\ell_{2}\right),
$$

and the missing transverse momentum 2-vector, $\not \not_{\mathrm{T}}$. Taking $x_{i}$ to be the momentum fractions carried away from the decay by $\ell_{i}, i=1,2$, we have

$$
\left(\frac{1}{x_{1}}-1\right) \mathbf{p}_{\mathbf{T}}\left(\ell_{1}\right)+\left(\frac{1}{x_{2}}-1\right) \mathbf{p}_{\mathbf{T}}\left(\ell_{2}\right)=\not p_{\mathbf{T}} .
$$

This yields two equations for $x_{1}$ and $x_{2}$ that can be solved to reconstruct the two original $\tau$ 4-momenta $p_{\tau}^{\mu}=p_{1}^{\mu} / x_{1}$, $p_{2}^{\mu} / x_{2}$. Thus, $M_{h}^{2}=\left(p_{1} / x_{1}+p_{2} / x_{2}\right)^{2}$, where $p_{1}=p\left(\ell_{1}\right)$ and $p_{2}=p\left(\ell_{2}\right)$. Physically, $x_{i}$ is constrained to $0<$ $x_{i}<1, i=1,2$, which reduces the background. Figure 1 presents the invariant mass distributions $M_{\text {col }}(\tau \tau)$ and $M_{\text {col }}(c, \tau \tau)$, which have sharp peaks near the Higgs boson and top-quark masses, respectively. We require the reconstructed Higgs boson mass and top-quark mass to lie in the mass windows $\Delta M_{\tau \tau}=0.20 M_{h}$ and $\Delta M_{c \tau \tau}=0.25 m_{t}$ using the ATLAS mass resolution [65]. We note that improvements in the discovery potential are possible by reducing the $\tau$ pair mass resolution $\Delta M_{\tau \tau}$.

Furthermore, we employ the cluster transverse mass distributions for $e^{ \pm} \mu^{\mp}$ and $c e^{ \pm} \mu^{\mp}$ with missing transverse energy $\left(\mathscr{E}_{T}\right)$ from the neutrinos to confirm the Higgs boson mass and top-quark mass reconstruction. These distributions have broad peaks near $M_{h}$ and $m_{t}$, respectively, as the kinematic characteristic of $t \rightarrow c h^{0} \rightarrow c e^{ \pm} \mu^{\mp}+\mathbb{E}_{T}$. The cluster transverse mass [70] is defined as

$$
M_{T}^{2}\left(C, \mathbb{E}_{T}\right)=\left(\sqrt{p_{T}^{2}(C)+M_{C}^{2}}+\mathbb{E}_{T}\right)^{2}-\left(\vec{p}_{T}(C)+\vec{E}_{T}\right)^{2},
$$

where $C=\ell^{ \pm} \ell^{\mp}$ or $c \ell^{ \pm} \ell^{\mp}, p_{T}(\ell \ell)$ or $p_{T}(c \ell \ell)$ is the total transverse momentum of all of the visible particles, while $M_{\ell \ell}$ and $M_{c \ell \ell}$ are the invariant cluster masses.

In most cases, the physics background can be reduced and the statistical significance for the Higgs boson signal enhanced if we apply a suitable requirement on the cluster transverse mass distributions [41] $M_{T}\left(\ell \ell, \mathbb{E}_{T}\right)$ and $M_{T}\left(c \ell \ell, \mathbb{E}_{T}\right)$. We have found that the acceptance requirement on $M_{\tau \tau}$ and $M_{c \tau \tau}$ is much more effective than the mass requirement on the cluster transverse masses. After the mass selection on the collinear invariant mass, the effects of additional requirements on the cluster transverse mass are negligible.

\section{Centrality of missing transverse energy}

To further suppress the physics background, the authors of Refs. $[37,40]$ suggested the use of the centrality of the missing transverse energy $\left(C_{\mathrm{MET}}\right)$,

$$
C_{\mathrm{MET}}=(x+y) / \sqrt{x^{2}+y^{2}},
$$




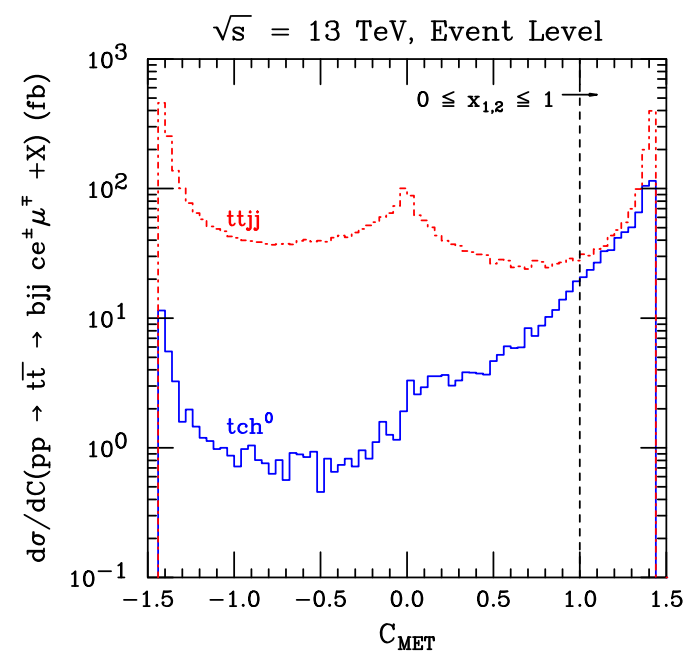

FIG. 2. Distribution for the centrality of missing transverse energy $\left(d \sigma / d C_{\mathrm{MET}}\right)$ for the FCNH signal from $t \rightarrow c h^{0} \rightarrow \tau \tau \rightarrow$ $e^{ \pm} \mu^{\mp}+\mathscr{E}_{T}$ (blue solid) at the LHC with $\sqrt{s}=13 \mathrm{TeV}$. Requiring the momentum fraction to be physical, $0 \leq x_{i} \leq 1, i=1,2$, we effectively select $C_{\mathrm{MET}}>1$, which is the region on the righthand side of the vertical dashed line. Also shown is the centrality distribution of the dominant physics background from $t t j j$ (red dotted dashed).

with

$$
x=\frac{\sin \left(\phi_{\mathrm{MET}}-\phi_{1}\right)}{\sin \left(\phi_{2}-\phi_{1}\right)}, \quad y=\frac{\sin \left(\phi_{2}-\phi_{\mathrm{MET}}\right)}{\sin \left(\phi_{2}-\phi_{1}\right)}
$$

where $\phi_{1,2}$ are the azimuthal angles of the two leptons ( $e$ or $\mu)$ in the transverse plane, and $\phi_{\mathrm{MET}}$ is the azimuthal angle of the transverse missing energy. Figure 2 shows the centrality $C_{\mathrm{MET}}$ for the FCNH signal from $t \rightarrow c h^{0}$ and the dominant background $t \bar{t} j j$. This is found to be less
TABLE I. $K$-factors at NLO for $t \bar{t} W$ and $t \bar{t} Z$ produced at the LHC.

\begin{tabular}{lccc}
\hline \hline Process $\backslash \sqrt{s}$ & $13 \mathrm{TeV}$ & $14 \mathrm{TeV}$ & $27 \mathrm{TeV}$ \\
\hline$t \bar{t} W$ & 1.64 & 1.66 & 1.70 \\
$t \bar{t} Z$ & 1.46 & 1.49 & 1.50 \\
\hline \hline
\end{tabular}

stringent than the requirement on the physical momentum fractions $0<x_{i}<1, i=1,2$, which leads to $C_{\mathrm{MET}}>1$.

\section{THE PHYSICS BACKGROUND}

The dominant background to the signal is from $t \bar{t} j j, j=$ $q$ or $g$. Here both top quarks decay leptonically $(t \rightarrow b \ell \nu)$ to the desired final-state combination of leptons. This comprises more than $95 \%$ of the total background. The other dominant contribution is from $p p \rightarrow b \bar{b} j j \tau \tau \rightarrow$ $b b j j e^{ \pm} \mu^{\mp}+E_{T}+X$ and $p p \rightarrow b \bar{b} j j W^{+} W^{-} \rightarrow b b j j e^{ \pm} \mu^{\mp}+$ $E_{T}+X$ (without a $t \bar{t}$ contribution) as well as $t \bar{t} W^{ \pm}$and $t \bar{t} Z$. For all of the backgrounds, one $b$ jet is selected while the other $b$ jet is misidentified as a light jet. Events with two $b$ jets having $p_{T}(b)>20 \mathrm{GeV}$ and $|\eta(b)|<2.5$ are vetoed [71,72]. We calculate the cross section for each of the backgrounds separately using MadGraph and apply the same event selection procedure as for the signal. The irreducible background from $p p \rightarrow t \bar{t} Z+X$ and $p p \rightarrow$ $t \bar{t} h^{0}+X$ with the subsequent decay of $Z \rightarrow \tau^{+} \tau^{-}$and $h^{0} \rightarrow$ $\tau^{+} \tau^{-}$is negligible after all acceptance requirements and the two- $b$ veto are imposed.

We scale our backgrounds to next-to-leading order (NLO) using $K$-factors of 1.8 for $t \bar{t}+2 j, b \bar{b} j j \tau \tau$, and $b \bar{b} j j W^{+} W^{-}$for all energies, i.e., $\sqrt{s}=13,14$, and $27 \mathrm{TeV}$. For $t \bar{t} W$ and $t \bar{t} Z$ we use the $K$-factors presented in Table I calculated with MadGraph5-aMC-NLO.
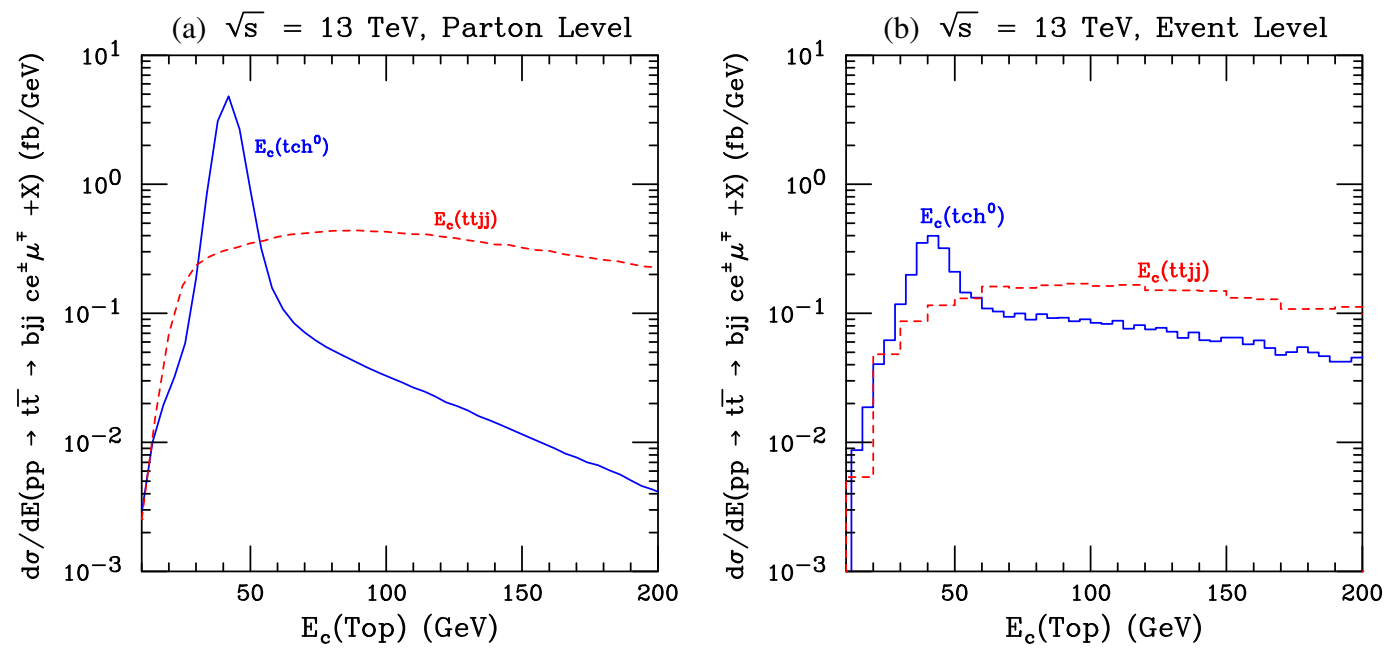

FIG. 3. Energy distribution for the charm quark $\left(d \sigma / d E_{c}\right)$ in the top rest frame for the Higgs signal in $p p$ collisions with $\sqrt{s}=13 \mathrm{TeV}$, from $t \rightarrow c h^{0}$ (blue solid) at the (a) parton level and (b) event level with detector simulations. Also shown is the charmquark energy distribution for the dominant background $t t j j$ (red dashed). 
After applying the event acceptance criteria, we reconstruct the invariant mass variables $M_{j_{1} j_{2}}, M_{b j_{1} j_{2}}, M_{\tau \tau}$, and $M_{c \tau \tau}$, as discussed in the previous section. In addition, the energy of the charm quark $\left(E_{c}\right)$ in the rest frame of the top quark is reconstructed to discriminate the $t \rightarrow c h^{0}$ signal from background [32,58]. For the flavor-changing top decay of $t \rightarrow c h^{0}$, the $E_{c}$ distribution exhibits a peak at the following value:

$$
E_{c}^{*}=\frac{m_{t}}{2}\left[1+\frac{m_{c}^{2}}{m_{t}^{2}}-\frac{m_{h}^{2}}{m_{t}^{2}}\right] \approx 41.43 \mathrm{GeV} .
$$

Requiring $29 \mathrm{GeV}<E_{c}<54 \mathrm{GeV}$, the background is significantly reduced while most of the signal is maintained. Figure 3 presents the energy distributions of the charm quark in the top-quark rest frame.

From the invariant mass and the charm-quark energy distributions at the parton and event levels, the following mass requirements are deduced:

(i) $\left|M\left(j_{1}, j_{2}\right)-m_{W}\right| \leq 0.20 \times m_{W}$ and $\left|M\left(b, j_{1}, j_{2}\right)-m_{t}\right| \leq$ $0.25 \times m_{t}$

(ii) $\left|M_{\mathrm{col}}(\tau, \tau)-m_{h}\right| \leq 0.20 \times m_{h}$ and $\left|M_{\mathrm{col}}(c, \tau, \tau)-m_{t}\right| \leq$ $0.25 \times m_{t}$

(iii) $40 \mathrm{GeV} \leq M_{T}\left(\ell, \ell, \mathbb{E}_{T}\right) \leq 140 \mathrm{GeV}$ and $80 \mathrm{GeV} \leq$ $M_{T}\left(c, \ell, \ell, E_{T}\right) \leq 180 \mathrm{GeV}$, and

(iv) $29 \mathrm{GeV} \leq E_{c} \leq 54 \mathrm{GeV}$.

These requirements are chosen to remove the physics background in a manner that maximizes the statistical significance of the FCNH signal.

\section{DISCOVERY POTENTIAL AT THE PARTON LEVEL}

Applying all of the selection criteria at the parton level for $\sqrt{s}=13,14$ and $27 \mathrm{TeV}$, our signal cross sections for $\lambda_{\text {tch }}=0.064$ are shown in Table II. The cross sections with $\lambda_{t c h}=0.01$ are also presented for a simple estimate to find the cross sections for other values of this $\mathrm{FCNH}$ Yukawa coupling. The cross sections for the backgrounds

TABLE II. Signal cross section in fb after all cuts, scaled with $b$ tagging $=0.7$.

\begin{tabular}{lcc}
\hline \hline$\sqrt{s}(\mathrm{TeV})$ & $\lambda_{\text {tch }}=0.01$ & $\lambda_{\text {tch }}=0.064$ \\
\hline 13 & 0.0096 & 0.39 \\
14 & 0.012 & 0.46 \\
27 & 0.043 & 1.72 \\
\hline \hline
\end{tabular}

after applying the selection requirements are presented in Table III.

Figure 4 presents the estimated statistical significance $\left(N_{S S}\right)$ as a function of $\lambda_{t c h} / \sqrt{2}$ for the parton-level analysis, where $N_{S S}$ is calculated using [73]

$N_{S S}=\sqrt{2 \times\left(N_{S}+N_{B}\right) \ln \left(1+N_{S} / N_{B}\right)-2 \times N_{S}}$.

Here $N_{S}$ and $N_{B}$ are the number of signal and background events, respectively.

Table IV presents a comparison between this study and our previous study for $t \rightarrow c h^{0} \rightarrow c W W^{*} \rightarrow c e^{ \pm} \mu^{\mp}+\mathbb{E}_{T}$ [41]. This analysis suggests that $h^{0} \rightarrow \tau \tau$ is much cleaner, because the Higgs boson mass is fully reconstructed and the energy of the charm quark in the top-quark rest frame improves the statistical significance using the optimized requirements.

Figure 5 presents the $5 \sigma$ discovery reach at the LHC for (a) $\sqrt{s}=14 \mathrm{TeV}$ and (b) $\sqrt{s}=27 \mathrm{TeV}$ at the parton level in the $\left[\cos (\beta-\alpha), \tilde{\rho}_{t c}\right]$ plane. We have chosen $\mathcal{L}=300$ and $3000 \mathrm{fb}^{-1}$. It is clear that the high-energy LHC at $\sqrt{s}=27 \mathrm{TeV}$ with a high luminosity $L=3000 \mathrm{fb}^{-1}$ significantly improves the discovery potential of $t \rightarrow c h^{0}$ for $\lambda_{\text {tch }} \geq 0.038$ beyond the current ATLAS limit [40] $\lambda_{\text {tch }}=0.064$.

\section{EVENT-LEVEL ANALYSIS WITH BOOSTED DECISION TREES}

In this section, we present the event-level analysis using the event generator Pythia 8 [54] and the detector simulation program Delphes [55]. From this analysis, the cross sections for the FCNH signal and the backgrounds are shown in Table $\mathrm{V}$ after applying the selection requirements.

For the event-level analysis, the mass resolutions are worse than at the parton level. Therefore, the mass selection window is relaxed and the selected events are used to train and test the boosted decision tree (BDT) classifier to increase the background rejection relative to signal acceptance. The Root [74] TMVA [75] package is used to perform the signal and background classification. We apply the following requirements to the sample:

(i) $65 \mathrm{GeV} \leq M\left(j_{1}, j_{2}\right) \leq 100 \mathrm{GeV}$,

(ii) $40 \mathrm{GeV} \leq M_{T}(\ell, \ell) \leq 300 \mathrm{GeV}$,

(iii) $M_{\mathrm{col}}(\tau, \tau) \leq 200 \mathrm{GeV}$ and $M_{\mathrm{col}}(c, \tau, \tau) \leq 300 \mathrm{GeV}$, and

(iv) $20 \mathrm{GeV} \leq E_{c} \leq 70 \mathrm{GeV}$.

TABLE III. Background cross sections in fb after applying the mass selection at the parton level.

\begin{tabular}{lccccccc}
\hline \hline$\sqrt{s}(\mathrm{TeV})$ & $t \bar{t} j j$ & $t \bar{t} j j(+\tau)$ one $t \rightarrow b \tau \nu$ & $b \bar{b} j j \tau \tau$ & $b \bar{b} j j W W$ & $t \bar{t} j j(+\tau \tau) t \bar{t} \rightarrow b \bar{b} \tau^{+} \tau^{-} \nu \nu$ & $t \bar{t} \mathrm{~V}$ & Total \\
\hline 13 & 0.45 & 0.21 & 0.021 & $3.2 \times 10^{-4}$ & 0.012 & $3.5 \times 10^{-3}$ & 0.68 \\
14 & 0.52 & 0.25 & 0.025 & $3.8 \times 10^{-4}$ & 0.014 & $3.8 \times 10^{-4}$ & 0.8 \\
27 & 1.96 & 0.9 & 0.074 & $1.3 \times 10^{-3}$ & 0.05 & $9.8 \times 10^{-3}$ & 2.99 \\
\hline \hline
\end{tabular}


TABLE IV. Minimum $\lambda_{\text {tch }}$ at $\mathcal{L}=3000 \mathrm{fb}^{-1}$ for $5 \sigma$.

\begin{tabular}{lcc}
\hline \hline$\sqrt{s}(\mathrm{TeV})$ & $h^{0} \rightarrow W W^{*}$ & $h^{0} \rightarrow \tau^{+} \tau^{-}$ \\
\hline 13 & 0.060 & 0.033 \\
14 & 0.057 & 0.031 \\
27 & 0.041 & 0.023 \\
\hline \hline
\end{tabular}

We then process it through the BDT, which contains 1000 trees at a depth of 5. The BDT response is shown in Fig 6. The BDT is employed to optimize the selection requirements and improve the statistical significance.

Event selection using the BDT classifier improves the statistical significance of the analysis relative to using an event-based selection on kinematic and acceptance variables only. Table VI shows that the BDT analysis improves the statistical significance by more than a factor of 2 .
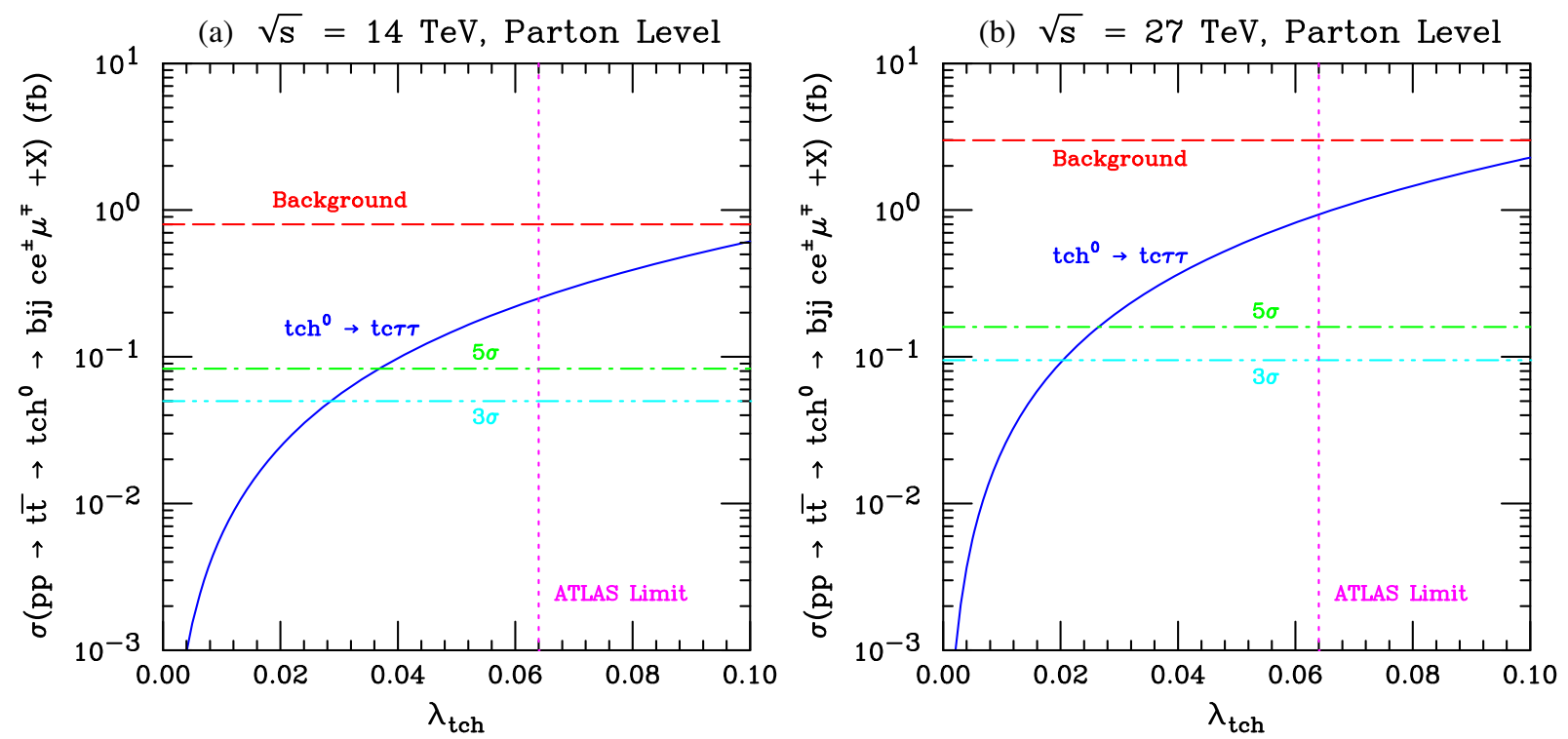

FIG. 4. Cross section of $p p \rightarrow t c h^{0} \rightarrow t c \tau \tau \rightarrow b j j c e^{ \pm} \mu^{\mp}+E_{T}+X$ (blue solid) in fb as a function of $\lambda_{t c h}$ for $\sqrt{s}=($ a) 14 TeV and (b) $27 \mathrm{TeV}$. Also shown are the cross section required for $3 \sigma$ (cyan dotted dashed) and $5 \sigma$ (green dotted dashed) as well as the cross section of the physics background (red dashed).

(a) $\sqrt{s}=14 \mathrm{TeV}$, Parton Level

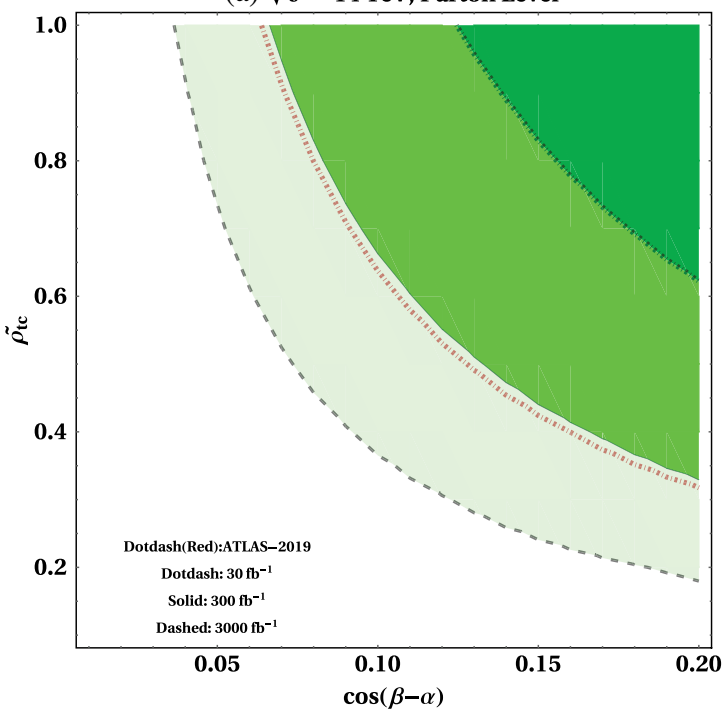

(b) $\sqrt{s}=27$ TeV, Parton Level

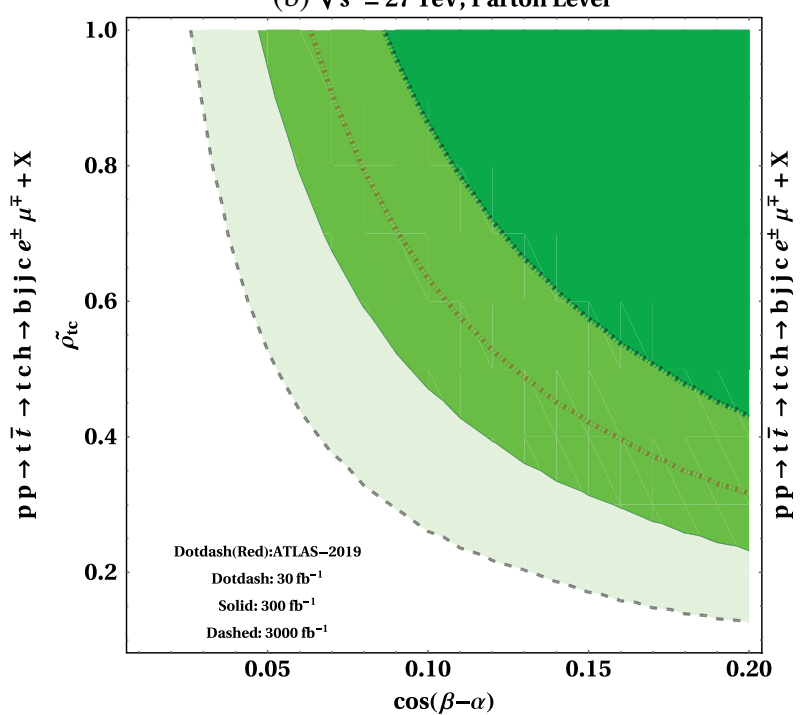

FIG. 5. Parton-level $5 \sigma$ discovery contours at the LHC in the $\left[\cos (\beta-\alpha), \tilde{\rho}_{t c}\right]$ plane for (a) $\sqrt{s}=14 \mathrm{TeV}$ and (b) $\sqrt{s}=27 \mathrm{TeV}$ with $L=30 \mathrm{fb}^{-1}$ (dark green dotted dashed), $300 \mathrm{fb}^{-1}$ (medium green solid) and $3000 \mathrm{fb}^{-1}$ (light green dashed) Also shown is the current limit on $\lambda_{t c h}=\tilde{\rho}_{t c} \cos (\beta-\alpha)$ (red dotted dashed) set by ATLAS [40]. 
TABLE V. Event-level cross section of signal and backgrounds in $\mathrm{fb}$ at the LHC with $\sqrt{s}=13 \mathrm{TeV}$ and all selection requirements.

\begin{tabular}{lc}
\hline \hline Process & Cross section \\
\hline$t \bar{t} j j$ & 1.30 \\
$b \bar{b} j j \tau \tau$ & 0.07 \\
$t \bar{t} W$ & 0.008 \\
$t \bar{t} Z$ & 0.001 \\
$t \bar{t} h^{0}$ & 0.0002 \\
$b \bar{b} j j W W$ & 0.001 \\
Total background & $\approx 1.4$ \\
Signal $\left(\lambda_{t c h}=0.01\right)$ & 0.00098 \\
Signal $\left(\lambda_{t c h}=0.064\right)$ & 0.040 \\
\hline \hline
\end{tabular}

Table VII presents the $95 \%$ confidence level limits on $\lambda_{\text {tch }}$ at $\sqrt{s}=13,14$ and $27 \mathrm{TeV}$ using an integrated $\mathcal{L}=300$ and $3000 \mathrm{fb}^{-1}$. In addition, the minimum $\lambda_{\text {tch }}$ for $5 \sigma$ discovery at the LHC is presented in Table VIII. We conclude that it will be difficult to discover this channel at 13 and $14 \mathrm{TeV}$ colliders in this channel, but a $27 \mathrm{TeV}$ high-energy collider holds promise for this signature.

Figure 7 presents the $5 \sigma$ discovery reach at the LHC for (a) $\sqrt{s}=14 \mathrm{TeV}$ and (b) $\sqrt{s}=27 \mathrm{TeV}$ at the event level in the $\left[\cos (\beta-\alpha), \tilde{\rho}_{t c}\right]$ plane. We have chosen $\mathcal{L}=300$ and $3000 \mathrm{fb}^{-1}$. It is clear that the high-energy LHC at $\sqrt{s}=$ $27 \mathrm{TeV}$ with a high luminosity $L=3000 \mathrm{fb}^{-1}$ significantly improves the discovery potential of $t \rightarrow c h^{0}$ beyond the current ATLAS limit [40] $\lambda_{t c h}=0.064$.

We have illustrated the improvement in statistical significance achieved by using a boosted decision tree classifier relative to a cut-based analysis. To avoid overtraining the BDT due to low statistics in the event-level analysis, the more restrictive parton-level invariant mass

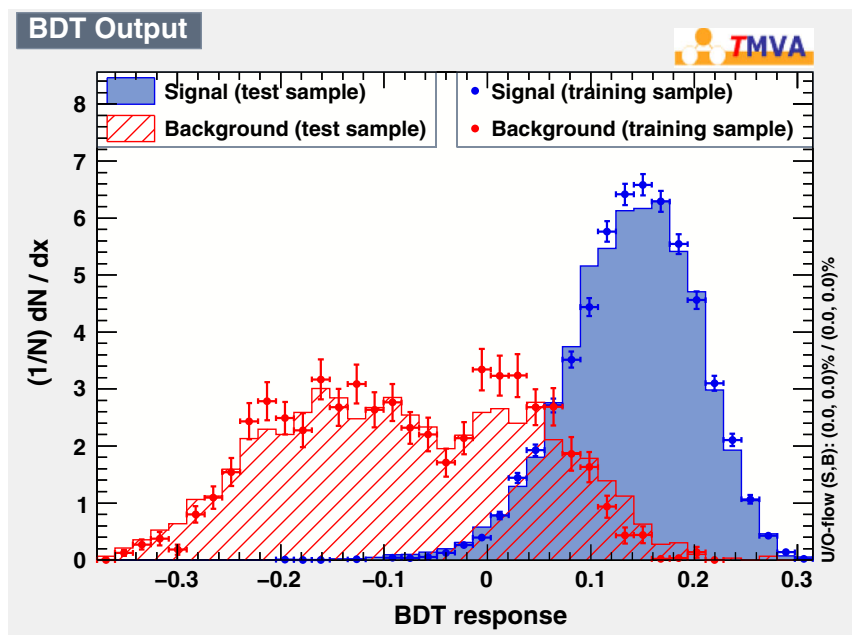

FIG. 6. BDT response for the signal of $t \rightarrow c h^{0}$ and the physics background at the event level.
TABLE VI. A comparison of the statistical significance at $\lambda_{\text {tch }} \approx 0.064$ and $\mathcal{L}=3000 \mathrm{fb}^{-1}$ between a kinematic variable selection analysis (Cut-Based) and BDT analysis.

\begin{tabular}{lcc}
\hline \hline$\sqrt{s}(\mathrm{TeV})$ & Cut-Based & BDT \\
\hline 13 & 1.2 & 2.7 \\
14 & 1.3 & 3.2 \\
27 & 2.2 & 5.5 \\
\hline \hline
\end{tabular}

TABLE VII. 95\% C.L. limits on $\lambda_{\text {tch }}$ at different collider energies and integrated luminosities.

\begin{tabular}{lcc}
\hline \hline$\sqrt{s}(\mathrm{TeV})$ & $\mathcal{L}=300 \mathrm{fb}^{-1}$ & $\mathcal{L}=3000 \mathrm{fb}^{-1}$ \\
\hline 13 & 0.099 & 0.055 \\
14 & 0.092 & 0.051 \\
27 & 0.068 & 0.038 \\
\hline \hline
\end{tabular}

TABLE VIII. Minimal $\lambda_{t c h}$ for $5 \sigma$ discovery at different collider energies and integrated luminosities.

\begin{tabular}{lcc}
\hline \hline$\sqrt{s}(\mathrm{TeV})$ & $\mathcal{L}=300 \mathrm{fb}^{-1}$ & $\mathcal{L}=3000 \mathrm{fb}^{-1}$ \\
\hline 13 & 0.21 & 0.088 \\
14 & 0.16 & 0.082 \\
27 & 0.11 & 0.061 \\
\hline \hline
\end{tabular}

requirements are relaxed. We then rely on the BDT to optimize the selections on the kinematic variables. Our goal is to improve the significance by using the BDT to set the requirements on the invariant masses and the charm-quark energy, which is a strong signal-to-background discriminant. We encourage our experimental colleagues to include the charm-quark energy as an effective discriminant to further improve the potential of detecting this FCNH signature at the LHC.

\section{CONCLUSIONS}

Many beyond-the-Standard-Model theories contain tree-level contributions to FCNH interactions, especially for the third-generation fermions. These contributions arise naturally in models with additional Higgs doublets, such as the special two-Higgs-doublet model for the top quark [76], or a general $2 \mathrm{HDM}[45,46]$. In the decoupling [50] or alignment limits [51,52], the light Higgs boson $\left(h^{0}\right)$ resembles the Standard Model Higgs boson with a mass less than that of the top quark. This could engender the rare decay $t \rightarrow c h^{0}$.

We investigated the prospects for such a discovery at the LHC, focusing on the $t \bar{t}$ production channel and their subsequent decay, where one decays hadronically and the other decays through the FCNH mode. The primary background for this signal is $t \bar{t} j j$ with both top quarks decaying leptonically. This background contains one $b$ jet 
(a) $\sqrt{s}=14 \mathrm{TeV}$, Event Level

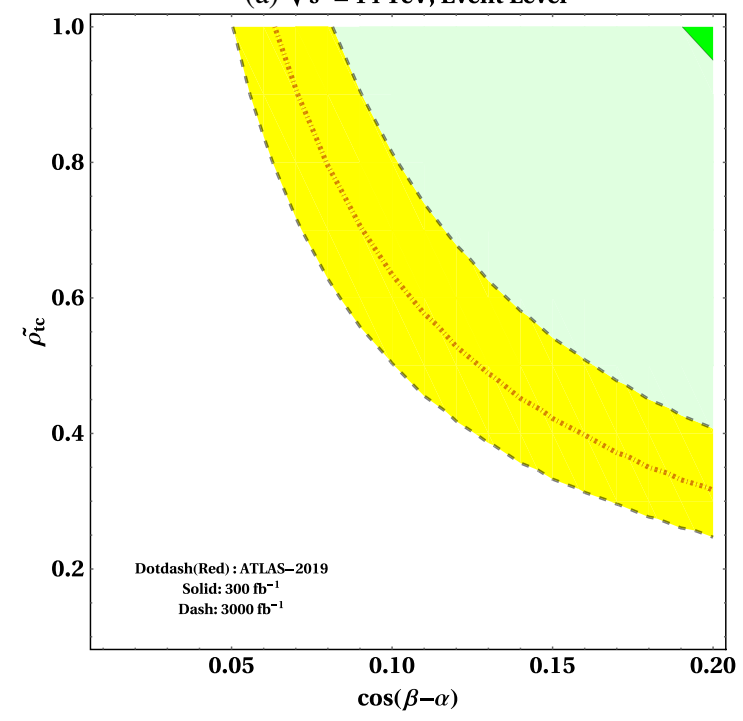

(b) $\sqrt{s}=27 \mathrm{TeV}$, Event Level

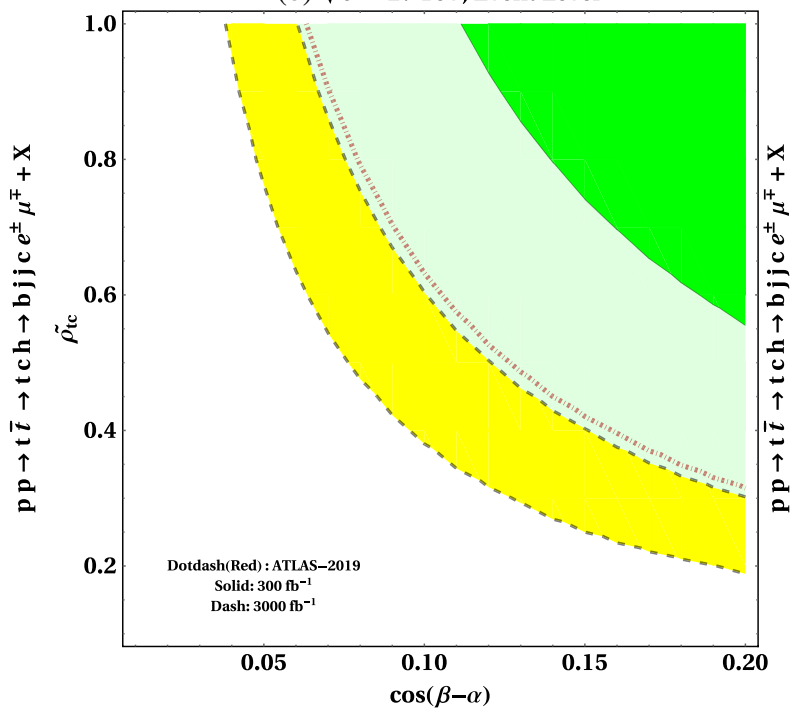

FIG. 7. Event-level $5 \sigma$ discovery contours at the LHC in the $\left[\cos (\beta-\alpha), \tilde{\rho}_{t c}\right]$ plane for (a) $\sqrt{s}=14 \mathrm{TeV}$ and (b) $\sqrt{s}=27 \mathrm{TeV}$ with $L=300 \mathrm{fb}^{-1}$ (medium green solid) and $3000 \mathrm{fb}^{-1}$ (light green dashed), as well as the event-level discovery contours and $3 \sigma$ contour (yellow dashed) Also shown is the current limit on $\lambda_{t c h}=\tilde{\rho}_{t c} \cos (\beta-\alpha)$ (red dotted dashed) set by ATLAS [40].

misidentified as a $c$ jet and two additional light jets, along with two leptons and missing transverse energy. Taking advantage of the available kinematic information, the $h^{0}$ and top-quark masses in the signal can be reconstructed and much of the background rejected.

Based on our parton-level analysis, we found that the LHC at $\sqrt{s}=14 \mathrm{TeV}$, with $\mathcal{L}=3000 \mathrm{fb}^{-1}$, can probe to as low as $\mathcal{B}\left(t \rightarrow c h^{0}\right) \approx 2.5 \times 10^{-4}$ with $\lambda_{\text {tch }} \approx 0.033$. At $\sqrt{s}=27 \mathrm{TeV}$, the reach is $\mathcal{B}\left(t \rightarrow c h^{0}\right) \approx 1.4 \times 10^{-4}$ with $\lambda_{\text {tch }} \approx 0.023$. The event-level analysis implies that there are technical challenges to reach the discovery potential of the parton-level analysis, especially improving efficiencies and mass reconstruction with high precision for final states with missing transverse energy from neutrinos.

In summary, we have made several significant contributions to searches for charming top decays with an associated Higgs boson:

(i) The $t \rightarrow c h^{0} \rightarrow \tau^{+} \tau^{-} \rightarrow c e^{ \pm} \mu^{\mp}$ has not been previously investigated as a dedicated discovery channel.

(ii) We demonstrated the effectiveness of reconstructing the Higgs boson and the top-quark masses by applying the collinear approximation to the tau decays.

(iii) We showed that the requirement on the momentum fractions $0 \leq x_{i} \leq 1, i=1,2$ is more effective at removing background and improving the significance than the requirement on centrality $\left(C_{\mathrm{MET}}>0\right)$. (iv) Our requirement on the energy of the charm quark $\left(E_{c}\right)$ in the top-quark rest frame significantly reduces the background and improves the significance.

(v) We have performed the first investigation of the discovery potential of $t \rightarrow c h^{0} \rightarrow \tau^{+} \tau^{-} \rightarrow c e^{ \pm} \mu^{\mp}$ for a high-energy $p p$ collider at $\sqrt{s}=27 \mathrm{TeV}$.

There are two useful features in the $\tau^{+} \tau^{-}$channel: (a) the reconstruction of $M_{h}$ and $m_{t}$ invariant masses by applying the collinear approximation, and (b) the selection requirement on the charm quark energy in the top-quark rest frame for reducing the physics background. This leads to the $\tau^{+} \tau^{-}$ discovery channel having a better reach in $\lambda_{t c h}$ by a factor of approximately 2 over the $W^{+} W^{-}$channel.

We look forward to being guided by new experimental results as we explore the interesting physics of EWSB and FCNH. While the properties of the Higgs boson undergo scrutiny as data is accumulated, dedicated FCNH searches for $t \rightarrow c h^{0}$ and $\phi^{0} \rightarrow t \bar{c}+\bar{t} c, \phi^{0}=H^{0}, A^{0}$ should be undertaken for the upcoming high-luminosity LHC and future high-energy $p p$ colliders.

\section{ACKNOWLEDGMENTS}

C. K. thanks George Hou and the High Energy Physics Group at National Taiwan University for excellent hospitality, where part of the research was completed. This research was supported in part by the U.S. Department of Energy. 
[1] G. Aad et al. (ATLAS Collaboration), Phys. Lett. B 716, 1 (2012).

[2] S. Chatrchyan et al. (CMS Collaboration), Phys. Lett. B 716, 30 (2012).

[3] A. M. Sirunyan et al. (CMS Collaboration), Phys. Rev. Lett. 125, 061801 (2020).

[4] G. Aad et al. (ATLAS Collaboration), Phys. Rev. Lett. 125, 051801 (2020).

[5] A. M. Sirunyan et al. (CMS Collaboration), Phys. Lett. B $\mathbf{8 0 5}, 135425$ (2020).

[6] G. Aad et al. (ATLAS Collaboration), J. High Energy Phys. 07 (2020) 108.

[7] A. D. Sakharov, Sov. Phys. Usp. 34, 392 (1991).

[8] G. W. Bennett et al. (Muon g-2 Collaboration), Phys. Rev. D 73, 072003 (2006).

[9] B. Abi et al. (Muon g-2 Collaboration), Phys. Rev. Lett. 126, 141801 (2021).

[10] S. Borsanyi, Z. Fodor, J. N. Guenther, C. Hoelbling, S. D. Katz, L. Lellouch, T. Lippert, K. Miura, L. Parato, K. K. Szabo et al., Nature (London) 593, 51 (2021).

[11] T. Aoyama, N. Asmussen, M. Benayoun, J. Bijnens, T. Blum, M. Bruno, I. Caprini, C. M. Carloni Calame, M. Cè, G. Colangelo et al., Phys. Rep. 887, 1 (2020).

[12] P. A. Zyla et al. (Particle Data Group), Prog. Theor. Exp. Phys. 2020, 083C01 (2020).

[13] J. P. Lees et al. (BABAR Collaboration), Phys. Rev. D 88, 072012 (2013).

[14] M. Huschle et al. (Belle Collaboration), Phys. Rev. D 92, 072014 (2015).

[15] R. Aaij et al. (LHCb Collaboration), Phys. Rev. Lett. 115, 111803 (2015); 115, 159901(E) (2015).

[16] R. Aaij et al. (LHCb Collaboration), J. High Energy Phys. 08 (2017) 055.

[17] A. M. Sirunyan et al. (CMS Collaboration), Phys. Lett. B 781, 517 (2018).

[18] A. Crivellin, C. Greub, and A. Kokulu, Phys. Rev. D 86, 054014 (2012).

[19] S. Weinberg, Phys. Rev. D 42, 860 (1990).

[20] K. Fuyuto, W. S. Hou, and E. Senaha, Phys. Lett. B 776, 402 (2018).

[21] W. S. Hou, Phys. Lett. B 296, 179 (1992).

[22] B. Altunkaynak, W. S. Hou, C. Kao, M. Kohda, and B. McCoy, Phys. Lett. B 751, 135 (2015).

[23] M. A. Arroyo-Ureña, A. Fernández-Téllez, and G. TavaresVelasco, arXiv:1906.07821.

[24] V. Khachatryan et al. (CMS Collaboration), Phys. Lett. B 749, 337 (2015).

[25] G. Aad et al. (ATLAS Collaboration), Eur. Phys. J. C 77, 70 (2017).

[26] A. M. Sirunyan et al. (CMS Collaboration), J. High Energy Phys. 06 (2018) 001.

[27] A. M. Sirunyan et al. (CMS Collaboration), J. High Energy Phys. 03 (2020) 103.

[28] J. A. Aguilar-Saavedra, Acta Phys. Pol. B 35, 2695 (2004).

[29] B. Mele, S. Petrarca, and A. Soddu, Phys. Lett. B 435, 401 (1998).

[30] G. Eilam, J. L. Hewett, and A. Soni, Phys. Rev. D 44, 1473 (1991); 59, 039901(E) (1998).

[31] J. A. Aguilar-Saavedra and G. C. Branco, Phys. Lett. B 495, 347 (2000).
[32] C. Kao, H. Y. Cheng, W. S. Hou, and J. Sayre, Phys. Lett. B 716, 225 (2012).

[33] K. F. Chen, W. S. Hou, C. Kao, and M. Kohda, Phys. Lett. B 725, 378 (2013).

[34] D. Atwood, S. K. Gupta, and A. Soni, J. High Energy Phys. 10 (2014) 057.

[35] V. Khachatryan et al. (CMS Collaboration), Phys. Rev. D 90, 112013 (2014).

[36] G. Durieux, F. Maltoni, and C. Zhang, Phys. Rev. D 91, 074017 (2015).

[37] X. Chen and L. Xia, Phys. Rev. D 93, 113010 (2016).

[38] V. Khachatryan et al. (CMS Collaboration), J. High Energy Phys. 02 (2017) 079.

[39] A. Papaefstathiou and G. Tetlalmatzi-Xolocotzi, Eur. Phys. J. C 78, 214 (2018).

[40] M. Aaboud et al. (ATLAS Collaboration), J. High Energy Phys. 05 (2019) 123.

[41] R. Jain and C. Kao, Phys. Rev. D 99, 055036 (2019).

[42] M. A. Arroyo-Ureña, R. Gaitán-Lozano, E. A. HerreraChacón, J. H. Y. Montes de Oca, and T. A. Valencia-Pérez, J. High Energy Phys. 07 (2019) 041.

[43] N. Castro, M. Chala, A. Peixoto, and M. Ramos, J. High Energy Phys. 10 (2020) 038.

[44] Y. J. Zhang and J. F. Shen, Eur. Phys. J. C 80, 811 (2020).

[45] S. Davidson and H.E. Haber, Phys. Rev. D 72, 035004 (2005); 72, 099902(E) (2005).

[46] F. Mahmoudi and O. Stal, Phys. Rev. D 81, 035016 (2010).

[47] J. F. Gunion, H. E. Haber, G. L. Kane, and S. Dawson, The Higgs Hunter's Guide (Addison-Wesley, Redwood City, 1990), ISBN 978-0201509359.

[48] A. M. Sirunyan et al. (CMS Collaboration), Eur. Phys. J. C 79, 421 (2019).

[49] G. Aad et al. (ATLAS Collaboration), Phys. Rev. D 101, 012002 (2020).

[50] J. F. Gunion and H.E. Haber, Phys. Rev. D 67, 075019 (2003).

[51] N. Craig, J. Galloway, and S. Thomas, arXiv:1305.2424.

[52] M. Carena, I. Low, N. R. Shah, and C. E. M. Wagner, J. High Energy Phys. 04 (2014) 015.

[53] ATLAS Collaboration, Report No. ATL-PHYS-PUB-2013012.

[54] T. Sjöstrand, S. Ask, J. R. Christiansen, R. Corke, N. Desai, P. Ilten, S. Mrenna, S. Prestel, C. O. Rasmussen, and P. Z. Skands, Comput. Phys. Commun. 191, 159 (2015).

[55] J. de Favereau, C. Delaere, P. Demin, A. Giammanco, V. Lemaître, A. Mertens, and M. Selvaggi (DELPHES 3 Collaboration), J. High Energy Phys. 02 (2014) 057.

[56] T. P. Cheng and M. Sher, Phys. Rev. D 35, 3484 (1987).

[57] K. Hagiwara, A. D. Martin, and D. Zeppenfeld, Phys. Lett. B 235, 198 (1990).

[58] T. Han, J. Jiang, and M. Sher, Phys. Lett. B 516, 337 (2001).

[59] J. Alwall, M. Herquet, F. Maltoni, O. Mattelaer, and T. Stelzer, J. High Energy Phys. 06 (2011) 128.

[60] S. Dulat, T. J. Hou, J. Gao, M. Guzzi, J. Huston, P. Nadolsky, J. Pumplin, C. Schmidt, D. Stump, and C. P. Yuan, Phys. Rev. D 93, 033006 (2016).

[61] M. Czakon and A. Mitov, Comput. Phys. Commun. 185, 2930 (2014).

[62] K. Hagiwara, T. Li, K. Mawatari, and J. Nakamura, Eur. Phys. J. C 73, 2489 (2013). 
[63] S. Hoeche, F. Krauss, N. Lavesson, L. Lonnblad, M. Mangano, A. Schalicke, and S. Schumann, 10.5170/CERN2005-014.288.

[64] G. Aad et al. (ATLAS Collaboration), Eur. Phys. J. C 79, 970 (2019).

[65] M. Aaboud et al. (ATLAS Collaboration), Phys. Rev. D 99, 072001 (2019).

[66] G. Aad et al. (ATLAS Collaboration), J. High Energy Phys. 11 (2019) 150.

[67] R. K. Ellis, I. Hinchliffe, M. Soldate, and J. J. van der Bij, Nucl. Phys. B297, 221 (1988).

[68] D. L. Rainwater, D. Zeppenfeld, and K. Hagiwara, Phys. Rev. D 59, 014037 (1998).

[69] T. Plehn, D. L. Rainwater, and D. Zeppenfeld, Phys. Rev. D 61, 093005 (2000).
[70] V.D. Barger and R. J. N. Phillips, Collider Physics (Addison-Wesley, Redwood City, 1987), ISBN 9780201058765.

[71] M. Aaboud et al. (ATLAS Collaboration), Eur. Phys. J. C 78, 186 (2018).

[72] A. M. Sirunyan et al. (CMS Collaboration), J. High Energy Phys. 10 (2018) 117.

[73] N. Kumar and S. P. Martin, Phys. Rev. D 92, 115018 (2015).

[74] R. Brun and F. Rademakers, Nucl. Instrum. Methods Phys. Res., Sect. A 389, 81 (1997).

[75] A. Hocker, P. Speckmayer, J. Stelzer, J. Therhaag, E. von Toerne, H. Voss, M. Backes, T. Carli, O. Cohen, A. Christov et al., arXiv:physics/0703039.

[76] A. K. Das and C. Kao, Phys. Lett. B 372, 106 (1996). 